\title{
Transformation of shoots into roots in Arabidopsis embryos mutant at the
} \section{TOPLESS locus}

\author{
Jeff A. Long ${ }^{1,3}$, Scott Woody ${ }^{1}$, Scott Poethig' ${ }^{2}$, Elliot M. Meyerowitz ${ }^{3}$ and M. Kathryn Barton ${ }^{1, *, \dagger}$ \\ ${ }^{1}$ Department of Genetics and Program in Cellular and Molecular Biology, University of Wisconsin-Madison, Madison, WI, USA \\ 2Department of Biology, University of Pennsylvania, Philadelphia, PA, USA \\ 3Division of Biology, California Institute of Technology, Pasadena, CA, USA \\ *Present address: Department of Plant Biology, Carnegie Institution of Washington, 260 Panama Street, Stanford, CA 94305, USA \\ †Author for correspondence (e-mail: barton@andrew2.stanford.edu) \\ Accepted 19 March 2002
}

\section{SUMMARY}

We describe a novel phenotype in Arabidopsis embryos homozygous for the temperature-sensitive topless-1 mutation. This mutation causes the transformation of the shoot pole into a root. Developing topless embryos fail to express markers for the shoot apical meristem (SHOOT MERISTEMLESS and UNUSUAL FLORALORGANS) and the hypocotyl (KNAT1). By contrast, the pattern of expression of root markers is either duplicated (LENNY, J1092) or expanded (SCARECROW). Shifts of developing topless embryos between permissive and restrictive temperatures show that apical fates (cotyledons plus shoot apical meristem) can be transformed to basal fates (root) as late as transition stage. As the apical pole of transition stage embryos shows both morphological and molecular characteristics of shoot development, this demonstrates that the topless 1 mutation is capable of causing structures specified as shoot to be respecified as root. Finally, our experiments fail to show a clear link between auxin signal transduction and topless-1 mutant activity: the development of the apical root in topless mutant individuals is not dependent on the activity of the predicted auxin response factor MONOPTEROS nor is the expression of DR5, a proposed 'auxin maximum reporter', expanded in the apical domain of topless embryos.

Key words: Arabidopsis, TOPLESS, Shoot transformation, Embryogenesis, Polarity

\section{INTRODUCTION}

Shoot and root systems of land plants carry out complementary functions fundamental to plant survival. These systems form from meristems established at opposite poles of the embryo. Morphological indications of differences along the shoot-root axis are most clearly manifested after the globular stage of embryogenesis. Globular stage embryos consist of two parts: a spherical mass of cells (the embryo proper) that gives rise to the seedling plant, and a suspensor, often filamentous, that anchors the embryo proper to the ovule and may act as a conduit for nutrients from the mother plant. In Arabidopsis, the suspensor consists of a single row of cells giving the globular stage embryo the appearance of a lollipop. In Arabidopsis, as in many other dicotyledonous plant species, the future shootroot axis of the globular embryo proper is aligned parallel to the long axis of the suspensor. The root pole forms from the side of the globular embryo closest to the suspensor, while the shoot pole forms from the side directly opposite. As the embryo makes the transition from the globular to the heart stage of embryogenesis, growth occurs more rapidly in the apical-basal dimension than in the radial dimension. Concomitant with this, morphological features of the shoot (cotyledons and shoot apical meristem) and root poles (organization of cell files and root cap) develop.

Polarity along the long axis of the globular stage embryo is foreshadowed in the polar organization of the egg cell and the zygote (Mansfield and Briarty, 1991; Mansfield et al., 1991). The egg cell is positioned in the embryo sac with the basal end facing the micropyle (the opening through which the pollen tube enters) and the apical end facing the central cell. The egg cell itself is polarized with the nucleus located closer to the apical end and the vacuole located closer to the basal end. The first division of the zygote is unequal, generating a large basal cell and a smaller terminal cell. The larger basal cell gives rise to the suspensor, while the smaller apical cell generates the embryo proper. The basal cell also gives rise to the hypophysis. The hypophysis is the only descendent of the basal cell to generate a portion of the seedling; it gives rise to portions of the root meristem and root cap (Mansfield and Briarty, 1991; Dolan et al., 1993).

Mutations in the GNOM/EMB30 gene disrupt both the polarity of the zygote and of the developing embryo proper (Mayer et al., 1993). The orientation of the first zygotic cell division is randomized in $g n$ mutant embryos and the most severely affected embryos develop as green balls that lack an axis. Polarity appears to be abolished at the cellular level in $g n$ 
mutants. Consistent with this, the PIN1 molecule, which is normally localized to the basal pole of the cell, is found around the circumference of the cell or localized to a side of the cell that bears no relationship to the apical-basal axis (Steinmann et al., 1999). The GN/EMB30 gene encodes a GEF/ARF-like molecule that may mediate its effects through targeted secretion (Shevell et al., 1994; Busch et al., 1996).

While polar cues embedded in the egg cell or early embryo may be important for early development of the embryo and for the proper alignment of the embryonic axis with that of the embryo sac, such cues are not absolutely required for the development of an embryo with a shoot-root axis. Ectopic embryos form from cells of the suspensor in some mutants (Vernon and Meinke, 1994). Embryos that form in this way may be oriented in the same or in the opposite direction to the embryo from which they derive. In addition, somatic embryos with a normal shoot-root axis can develop from a variety of tissue types.

Several mutants exist in which the development of the shoot or the root pole is disrupted, but in which a polar axis is nonetheless generated. For example, monopteros, bodenlos and axr6 mutations all interfere with the development of the root pole (Hardtke and Berleth, 1998; Hamann et al., 1999; Hobbie et al., 2000). Conversely, cup-shaped cotyledon (cuc) and shoot meristemless ( $\mathrm{stm}$ ) mutations interfere with the development of the cotyledons and meristem (Barton and Poethig, 1993; Aida et al., 1997). Such genes presumably act downstream of a signal that distinguishes cells of the future root pole from cells of the future shoot pole.

In cultured cells, ratios of the plant growth regulators cytokinin and auxin have been shown to play a role in the process of specifying cells as root or shoot (Skoog and Miller, 1957). High auxin to cytokinin ratios promote root development, while high cytokinin to auxin ratios promote shoot development. It is still unknown whether such ratios play a role in the embryo. However, the observation that axr6 mutants fail to develop a root and also fail to respond normally to auxin suggests they may (Hobbie et al., 2000).

One mutation, doppelwurzel, may cause a polar duplication of pattern during embryogenesis as it causes two complete seedlings to develop from a single ovule in a head to head (cotyledon to cotyledon) configuration (Jürgens et al., 1991). However, little is known about embryonic development in this mutant and it is possible that the apparent polar duplication is actually the result of the development of twin embryos from a single zygote.

We describe here a novel phenotype caused by the topless$l$ (tpl-1) mutation. In homozygous tpl-1 mutants the apical pole of the embryo develops as a root instead of as a shoot. The result is a seedling with two roots joined at the base and no hypocotyl, cotyledons or shoot apical meristem. The novel tpl1 mutation should provide an entrance to molecular genetic study of this important, but previously experimentally intractable, decision in plant development.

\section{MATERIALS AND METHODS}

\section{Genetics}

The tpl-1 mutation was isolated in the Landsberg erecta genetic background after EMS mutagenesis. Three backcrosses to Landsberg erecta were performed prior to phenotypic analysis. The tpl-1 mutation was mapped using CAPS markers as described by Konieczny and Ausubel (Konieczny and Ausubel, 1993). tpl-1 mutant individuals were crossed to wild-type Columbia individuals. The $\mathrm{F}_{1}$ progeny were allowed to self-fertilize at $24^{\circ} \mathrm{C}$. DNA was prepared from $\mathrm{F}_{2}$ seedlings exhibiting strong topless mutant phenotypes (monocot or more extreme). Three out of 268 chromosomes were recombinant between tpl-1 and CAPS marker g2395 (recombinant frequency $=1.1 \%$ ). Two out of 268 chromosomes were recombinant between $t p l-1$ and CAPS marker $\mathrm{m} 59$ (recombinant frequency $=0.8 \%$ ). In all cases, chromosomes recombinant between one marker and $t p l$ 1 were non-recombinant for the other marker, indicating that $t p l-1$ lies between them.

\section{In situ hybridization experiments}

In situ hybridization and probe preparation were performed as in Long and Barton (Long and Barton, 1998). The KNAT1, SCR, STM, ANT and $U F O$ probes have been described by Lincoln et al. (Lincoln et al., 1995), DiLaurenzio et al. (DiLaurenzio et al., 1996), Long et al. (Long et al., 1996), Long and Barton (Long and Barton, 1998) and Lee et al. (Lee et al., 1997), respectively.

\section{Histology}

Staining of $\beta$-glucuronidase (GUS) activity was performed by incubating embryos dissected from their ovules overnight at $37^{\circ} \mathrm{C}$ in $2 \mathrm{mM}$ 5-bromo-4-chloro-3-indolyl b-D-glucuronic acid, $100 \mathrm{mM}$ NaPo4, pH 7, 0.1\% Triton X-100, 1mM FeCN and 10 mM EDTA.
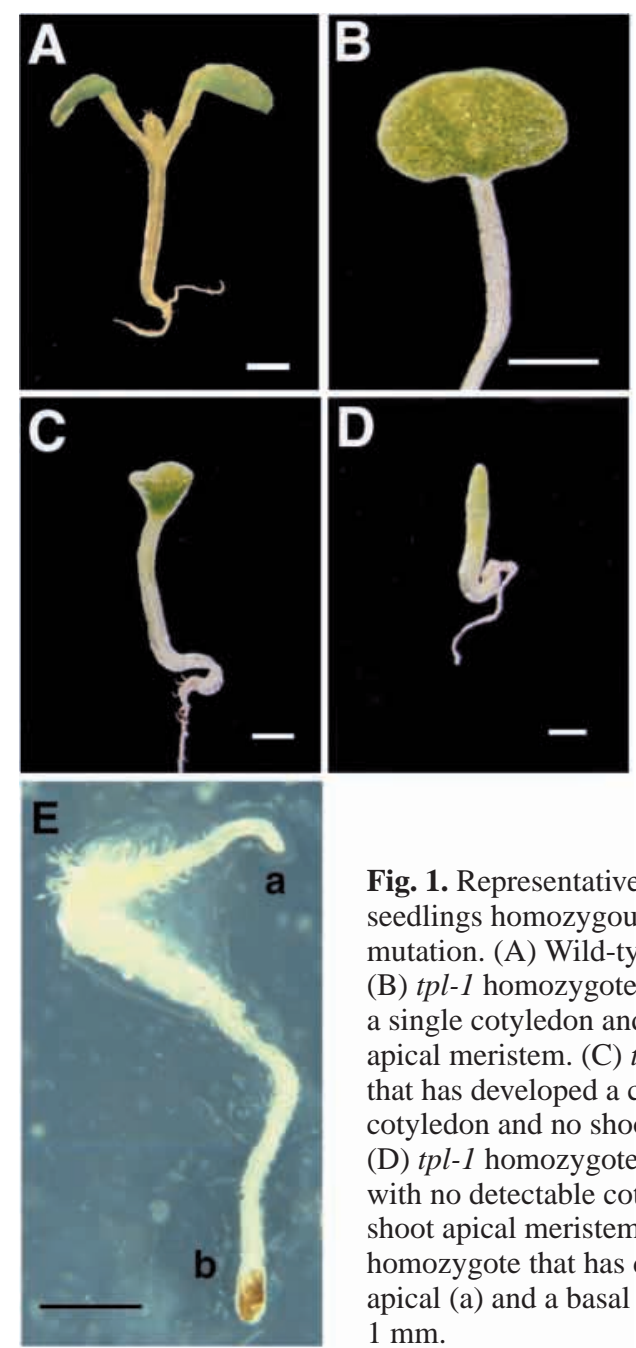

Fig. 1. Representative phenotypes of seedlings homozygous for the $t p l-1$ mutation. (A) Wild-type seedling. (B) tpl-1 homozygote that has developed a single cotyledon and lacks a shoot apical meristem. (C) tpl-1 homozygotes that has developed a cup-shaped cotyledon and no shoot apical meristem. (D) $t p l-1$ homozygote that has developed with no detectable cotyledon tissue or shoot apical meristem. (E) tpl-1 homozygote that has developed both an apical (a) and a basal (b) root. Scale bars: $1 \mathrm{~mm}$. 
Table 1. The topless-1 allele is temperature sensitive

\begin{tabular}{|c|c|c|c|c|}
\hline Temperature & One or two cotyledons, no SAM* & Tube-shaped cotyledon, no SAM & SAM and cotyledons absent & Shoot replaced by a root \\
\hline $24^{\circ} \mathrm{C}$ & $57 \%(n=254)$ & $36 \%(n=158)$ & $4 \%(n=17)$ & $3 \%(n=12)$ \\
\hline $28^{\circ} \mathrm{C}$ & $7 \%(n=25)$ & $16 \%(n=61)$ & $13 \%(n=49)$ & $64 \%(n=246)$ \\
\hline
\end{tabular}

*Absence of SAM refers to SAM formed during embryogenesis, not to postembryonic SAM formation. Nearly all seedlings of this class are able to form a SAM within 2 weeks of germination.

\section{Temperature shift experiments}

Homozygous tpl-1 plants were grown at $24^{\circ} \mathrm{C}$ until they began to flower. For each time point, 10-12 tpl-1 flowers were emasculated and allowed to mature at $24^{\circ} \mathrm{C}$ for 2 days. Pollen from tpl-1/tpl-1 plants was then crossed onto the carpels and the plants were immediately placed at either $29^{\circ} \mathrm{C}$ or $17^{\circ} \mathrm{C}$. To determine the developmental stage of the embryos being shifted, embryos from one hand pollination were dissected and examined microscopically at the time of each temperature shift.

\section{RESULTS}

\section{topless mutant phenotype - seedling stage}

Plants carrying the topless-1 (tpl-1) mutation were identified in a screen for seedlings defective in shoot apical meristem (SAM) formation (Barton and Poethig, 1993). The tpl-1 mutation maps to the upper arm of chromosome 1 between markers g2395 and m59 (see Materials and Methods for data).

When homozygous, the tpl-1 mutation causes either loss of apical structures or transformation of the apical pole of the embryo into a root. The tpl-1 mutation is temperature sensitive (Table 1). Lower temperatures increase the fraction of seedlings with cotyledon tissue and decrease the fraction of double-root seedlings. Conversely, higher temperatures increase the fraction of severely mutant seedlings, especially those with two roots.

Fig. 1B-D shows homozygous $t p l-1$ seedlings that fall into the 'loss' category. The mildest phenotype is a seedling with two cotyledons (fused to varying extents) and no SAM (not shown). Homozygous tpl- 1 seedlings may also develop a single cotyledon and no shoot apical meristem (Fig. 1B, Fig. 2B). More severely affected seedlings form a radially symmetric, cup-shaped cotyledon and lack a shoot apical meristem (Fig. 1C, Fig. 2C). Yet others appear to lack cotyledons and SAM completely, and only make a hypocotyl (Fig. 1D, Fig. 2D). (It is because of this last phenotype that the gene was named topless.)

Fig. 1E and Fig. 2E-F show homozygous tpl-1 seedlings that fall into the 'transformation' category. In these seedlings, an apical root replaces the shoot. The apical root has a root cap and exhibits normal gravitropism. The cellular organization of the apical root is roughly similar to a wild-type root with long files of cells converging near the root tip (Fig. 3). However, the apical root is often broader and appears to incorporate more cells than the wild-type basal root (Fig. 2F, Fig. 3).

Rarely, homozygous tpl-1 seedlings show simultaneous expression of apical and basal characteristics. Fig. $2 \mathrm{G}$ shows a homozygous tpl-1 seedling in which root hairs grow from a reduced cotyledon.

A fraction of topless mutant individuals are able to form a SAM by a few weeks after germination. These SAMs form at the adaxial base of the cotyledon. Such shoot apical meristems go on to form fairly normal, fertile plants which serve as a source of homozygous tpl-1 seed. The most penetrant abnormality detected in such tpl- 1 'escape' plants is a tendency for the carpels to be unfused (not shown). Nearly all tpl seedlings with one or two cotyledons are able to form SAMs postembryonically. topless mutants with cup-shaped cotyledons do so only rarely, while tpl-1 mutants in which the apical pole is transformed into a root have never been observed to form a SAM.

Seedlings heterozygous for tpl- 1 sometimes exhibit mild fusion of the cotyledons. To determine whether this is due to a dominant, zygotic effect of $t p l-1$ or to a maternal effect of
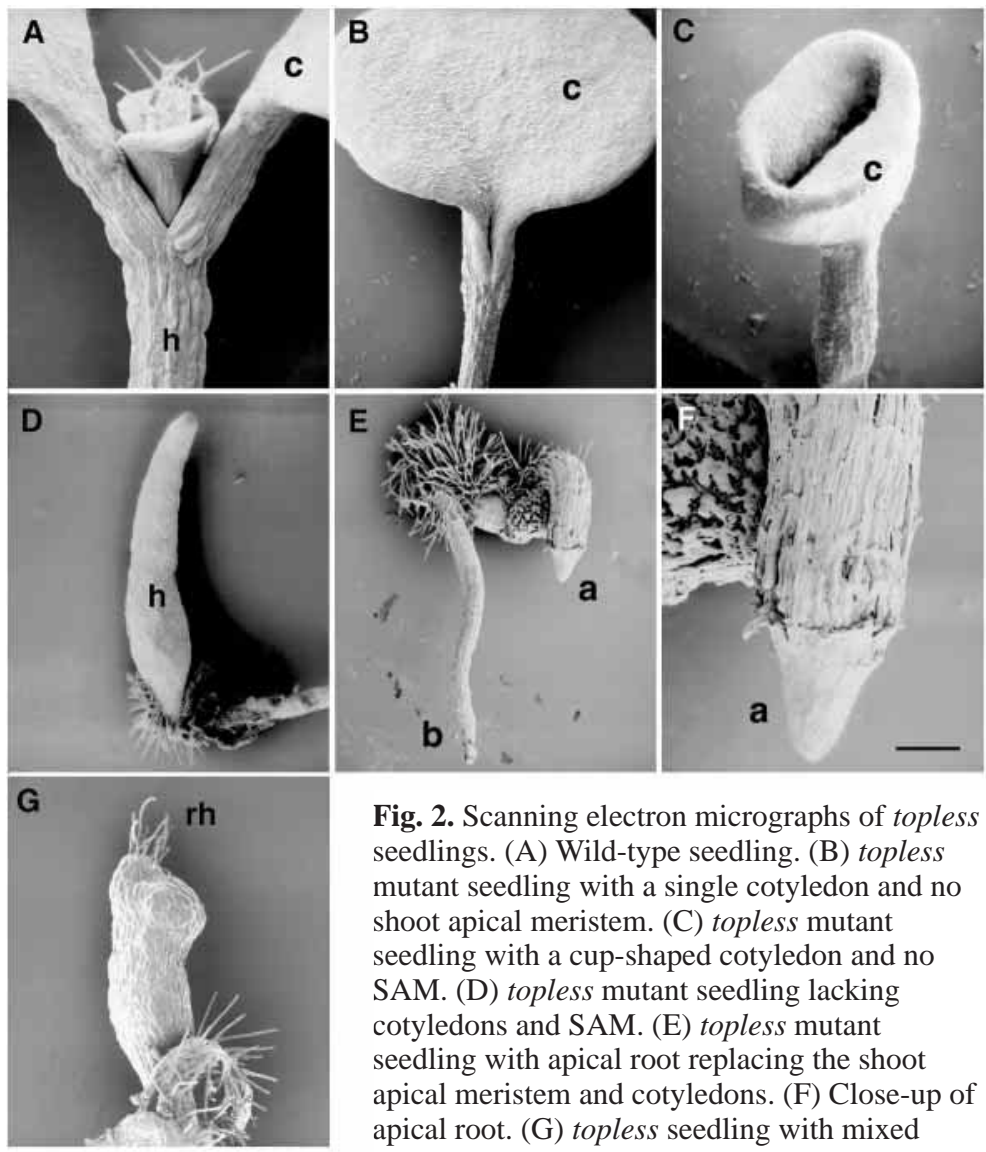

Fig. 2. Scanning electron micrographs of topless seedlings. (A) Wild-type seedling. (B) topless mutant seedling with a single cotyledon and no shoot apical meristem. (C) topless mutant seedling with a cup-shaped cotyledon and no SAM. (D) topless mutant seedling lacking cotyledons and SAM. (E) topless mutant seedling with apical root replacing the shoot apical meristem and cotyledons. (F) Close-up of apical root. $(\mathrm{G})$ topless seedling with mixed fates. Root hairs are present on a reduced cotyledon. h, hypocotyl; c, cotyledon; a, apical root; b, basal root; rh, root hairs. Scale bar: $0.2 \mathrm{~mm}$ in A and C; $0.37 \mathrm{~mm}$ in B and E; $0.43 \mathrm{~mm}$ in D and $\mathrm{G} ; 0.1 \mathrm{~mm}$ in $\mathrm{F}$. 


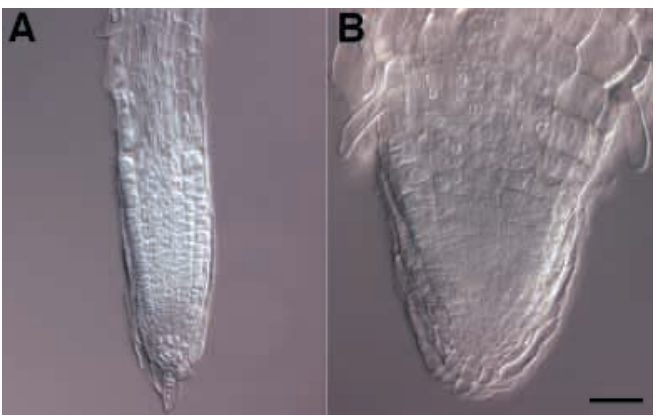

Fig. 3. DIC images of apical and basal topless roots. (A) Basal root. (B) Apical root. Scale bar: $0.1 \mathrm{~mm}$.

the tpl-1 mutation, reciprocal crosses were carried out. The fraction of heterozygotes showing a cotyledon fusion defect was the same whether the maternal or paternal parent was homozygous for tpl-1 (Table 2). Thus, there is no indication of a maternal effect in these experiments. Instead, we conclude the weak cotyledon fusion defect in $t p l-l$ heterozygotes is dependent on the genotype of the zygote and thus due to weak dominance of the $t p l-1$ allele.

\section{Development of topless mutant embryos}

tpl-1 mutant embryos are initially morphologically identical to wild-type embryos. At the globular stage they are similar to wild-type embryos in size, shape and cellular organization (see Fig. 5J). By the heart stage, tpl-1 mutant embryos differ from wild-type embryos. They are oblong and lack apparent cotyledon primordia (Fig. 4). The oblong tpl embryos continue to elongate during embryogenesis, accumulate storage proteins and mature.

To further determine the extent to which $t p l$ mutants deviate from wild-type embryos in early development, a panel of markers for apical and basal fates was examined in mutant embryos.

\section{Markers associated with apical fates}

Mutants homozygous for $t p l-1$ fail to form a SAM and frequently also lack cotyledons. These defects could be due to a failure to specify cells as SAM or cotyledon cells in the apical region of the globular embryo. Alternatively, they could be due to a failure of properly specified cells to develop into recognizable structures. To distinguish between these possibilities, we analyzed markers normally expressed in early SAM and cotyledon development in topless mutant embryos.

The SHOOTMERISTEMLESS (STM) gene is required for

Table 2. The tpl1 mutation is incompletely dominant

\begin{tabular}{lccc}
\hline $\begin{array}{l}\text { Temperature } \\
\left({ }^{\circ} \mathrm{C}\right)\end{array}$ & Female parent & Male parent & $\begin{array}{c}\text { Number of progeny } \\
\text { with fused cotyledons }\end{array}$ \\
\hline 29 & $T P L / T P L$ & $t p l / t p l$ & $86 / 390(22 \%)$ \\
& $t p l / t p l$ & $T P L / T P L$ & $100 / 429(23 \%)$ \\
24 & $T P L / T P L$ & $t p l / t p l$ & $4 / 195(2.1 \%)$ \\
& $t p l / t p l$ & $T P L / T P L$ & $3 / 178(1.7 \%)$ \\
17 & $T P L / T P L$ & $t p l / t p l$ & $2 / 144(1.4 \%)$ \\
& $t p l / t p l$ & $T P L / T P L$ & $3 / 135(2.2 \%)$ \\
\hline
\end{tabular}

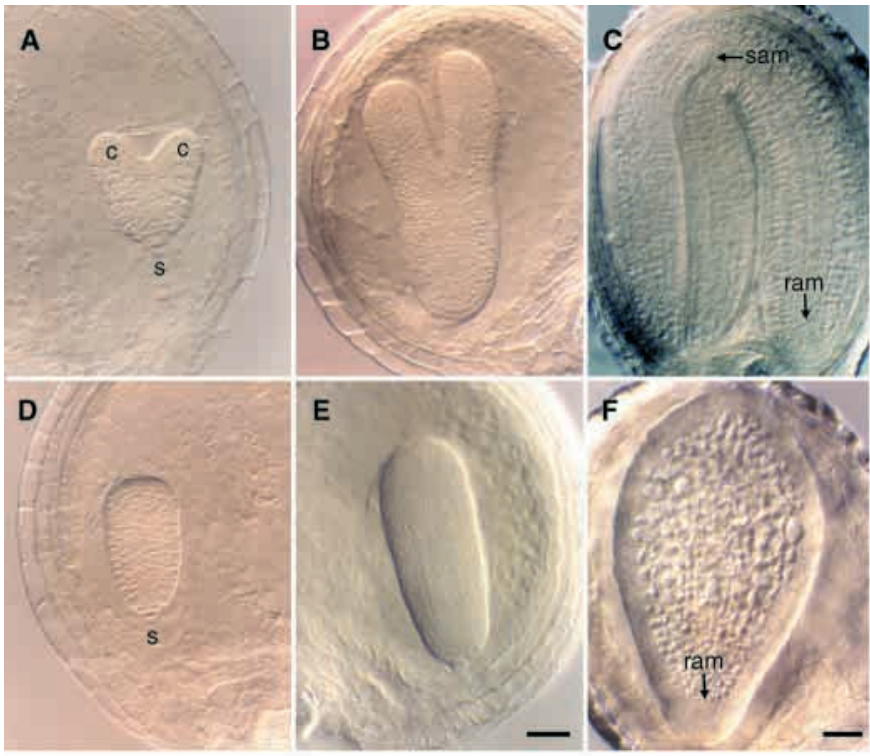

Fig. 4. DIC images of developing wild-type and topless embryos. All seedlings developed at $29^{\circ} \mathrm{C}$. (A) Wild-type heart stage embryo.

(B) Wild-type torpedo stage embryo. (C) Wild-type mature embryo. (D) topless heart stage embryo. (E) topless torpedo stage embryo. (F) topless mature embryo. c, cotyledon; s, suspensor; sam, shoot apical meristem; ram, root apical meristem. Scale bars: in E, $25 \mu \mathrm{m}$ for $\mathrm{A}, \mathrm{B}, \mathrm{D}, \mathrm{E}$; in $\mathrm{F}$ for $25 \mu \mathrm{m} \mathrm{C}, \mathrm{F}$.

initiation and maintenance of the SAM (Barton and Poethig, 1993; Long and Barton, 1998). In wild-type embryos, STM RNA marks the developing SAM and is first detected at the late globular stage, before there is morphological evidence of a shoot apical meristem. In homozygous tpl-1 embryos, the $S T M$ transcript is not detected $(n>50$; Fig. 5B). Wild-type siblings segregating in the same silique and processed along with the tpl-1 embryos exhibited normal STM expression (Fig. 5A). tpl-1 embryos accumulate transcripts related to cellular functions, such as the UBIQUITIN transcript, which could be detected throughout the $t p l-1$ embryo (Fig. 5D).

$U F O$ is also expressed in the SAM during embryogenesis. Accumulation of the $U F O$ transcript is dependent on wild-type $S T M$ function (Long and Barton, 1998). Given the failure of STM expression in tpl-1 mutants, we would expect $U F O$ transcript also to be missing. Indeed, $U F O$ mRNA fails to accumulate in tpl-1 mutants $(n>50$; Fig. 5C). As in the STM experiment, control wild-type embryos processed simultaneously showed normal $U F O$ expression (not shown).

Finally, the distribution of a transcript ordinarily found in the developing hypocotyl was examined. KNAT1 is normally expressed in peripheral regions of the hypocotyl (Fig. 5G). In tpl-1 mutants, KNAT1 transcript is either missing or only faintly expressed (Fig. 5H). Loss of KNAT1 transcript accumulation is somewhat surprising as $t p l-1$ mutant seedlings grown at $24^{\circ} \mathrm{C}$ usually make a structure recognizable as a hypocotyl. The expression data suggest that the program for hypocotyl development may be somewhat impaired even at this relatively permissive temperature.

The above experiments were done at $24^{\circ} \mathrm{C}$, a temperature that does not cause a particularly large number of double root seedlings (Table 1). The experiments have not been carried out 
at $29^{\circ} \mathrm{C}$. However, given that there is even less development of apical structures at $29^{\circ} \mathrm{C}$, it is unlikely that expression of $S T M$, $U F O$ or $K N A T 1$ would be restored at the higher temperature.

The experiments with the UFO, STM and KNATI markers indicate that the failure of apical structures to develop in $t p l-1$ mutants is due to a failure to specify apical (shoot) fates, rather than a failure of properly specified domains to grow and develop into morphologically recognizable structures.

The AINTEGUMENTA (ANT) RNA marks developing leaf, floral organ and cotyledon primordia (Elliott et al., 1996; Long and Barton, 1998). In the wild-type embryo $A N T$ is first expressed in the globular stage embryo prior to any morphological evidence of cotyledon initiation. The two cotyledon primordia appear to be specified initially as a single donut-shaped primordium encircling the apical half of the globular embryo (Fig. 5E). In tpl-1 mutants, by contrast, ANT transcript is found in the center of the apical pole of the embryo in addition to the peripheral ring $(n>200$; Fig. 5F). ANT transcript was also found in topless embryos developing at the most restrictive temperature $\left(29^{\circ} \mathrm{C}\right)$. However, at this temperature, it is often confined to a more apical region: the outermost (protodermal) layer (not shown).

The experiments with the ANT marker indicate that some cells are specified as cotyledon in tpl-1 mutants, even at the most restrictive temperature. However, the pattern of cells specified is altered: the altered pattern shows changes both in the apical/basal dimension of the embryo as well as in the radial (or central/peripheral) dimension.

\section{Markers associated with basal fates}

Homozygous tpl-1 mutant embryos developing at $29^{\circ} \mathrm{C}$ show a high frequency of apparent shoot to root transformation. To further investigate the development of such double root embryos, we analyzed the expression of three markers for root cell fates. The SCARECROW transcript accumulates in the lenticular cell and in the cortical cell files (Fig. 5I) (DiLaurenzio et al., 1996). In the wild-type embryo, $S C R$ expression is limited to the region below the $\mathrm{O}$ ' boundary. (The O' boundary divides the globular stage embryo into apical and basal halves. It is created when the four cells of the quadrant stage embryo divide to generate the octant stage embryo.) In $t p l-1$ mutant embryos, scr extends above the O' boundary, indicating that root and/or hypocotyl fates extend further apically in topless mutant embryos (Fig. 5J).

The $L E N N Y$ reporter was isolated in a screen of enhancer trap lines. [The approach used has been described by Sundaresan et al. (Sundaresan et al., 1995).] The $L E N N Y$ reporter expresses GUS activity in the root pole of the developing embryo and not in the shoot pole (Fig. 6A,B). LENNY is initially expressed in the lenticular cell (a descendent of the hypophysis) beginning at the heart stage. Expression later spreads to encompass surrounding cells in the root apical meristem.

Twenty-one percent (16/76) of homozygous topless mutant embryos developing at $29^{\circ} \mathrm{C}$ show expression of the $L E N N Y$ reporter at both poles of the embryo by 168 hours postfertilization (Fig. 6D-F). (Wild-type embryos grown at $29^{\circ} \mathrm{C}$ are typically at bent cotyledon stage by 168 hours.) The extent to which embryos express the $L E N N Y$ reporter varies. Some embryos show only a weak area of $L E N N Y$ expression at the apical pole (Fig. 6D). More commonly, both poles express LENNY with near equal intensity (Fig. 6E,F).

Note that the apical root primordium does not have the same cellular organization as the normal, basal, root primordium. For example, the files of cells that make up the developing vasculature are not apparent in the $t p l$ apical root (Fig. 6E,F). In addition, the apical root primordium is not tapered as in a normal root but instead is much wider.

A small fraction (less than 5\%) of $t p l$ embryos developing at $29^{\circ} \mathrm{C}$ arrest during embryogenesis. The arrested embryos express $L E N N Y$ throughout a greater fraction of the embryo than do the non-arrested embryos (Fig. 6G,H). One interpretation of this arrested phenotype is that it reflects an early and more complete transformation of apical to basal fates and that such a transformation results in the arrest of embryonic growth. However, it is also possible that a second, unrelated mutation is responsible for the arrest or that the topless mutation interferes with the viability of early embryos in a way that is unrelated to the specification of apical or basal fates.

The J1092 marker is expressed in the embryo in presumptive root cap cells (Fig. 6C) (described by J. Hasseloff, http://www.plantsci.cam.ac.uk/Haseloff/Home.html). Thirty seven percent $(15 / 41)$ of homozygous topless mutant embryos developing at $29^{\circ} \mathrm{C}$ show expression of the $\mathrm{J} 1092$ reporter at both poles of the embryo by 168 hours postfertilization (Fig. 6J,K).

In summary, markers for basal root fates were often found expressed in the region of the developing apical root of topless mutants. Taken together with the experiments with apical

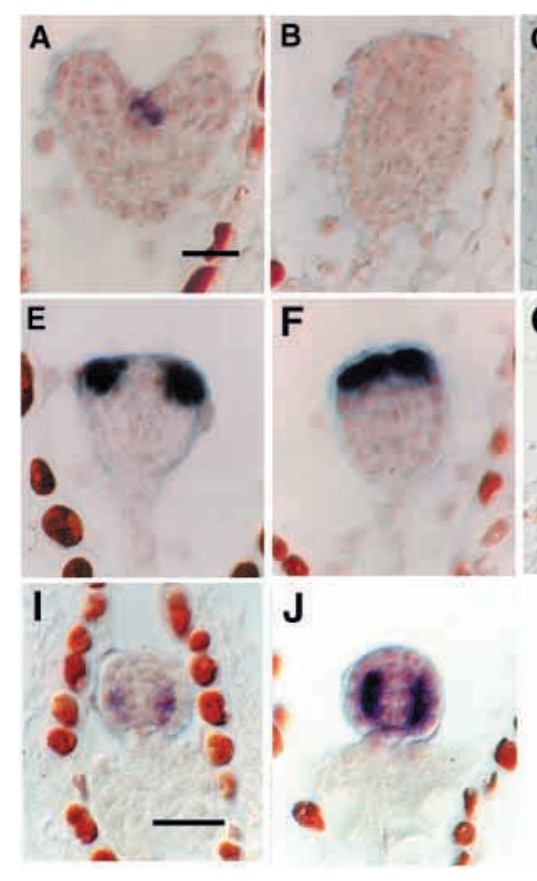

expression in topless mutant embryo. (E) ANT expression in wild-type heart stage embryo. (F) ANT expression in topless mutant. (G) KNAT1 expression in wild-type walking stick stage embryo. (H) KNAT1 expression in topless embryo. (I) SCARECROW expression in wild-type globular embryos. (J) SCARECROW expression in topless mutant embryos. Scale bars: in A, $25 \mu \mathrm{m}$ for A-F; in G, $50 \mu \mathrm{m}$ for G,H; in I, $25 \mu \mathrm{m}$ for I,J. 
markers, we conclude that there is a transformation of the apical region of the embryo from a shoot 'primordium' into a root 'primordium' in topless mutant embryos.

\section{Expression of the DR5 auxin reporter in topless mutants}

A high auxin to cytokinin ratio will promote root development over shoot development in cultured cells (Skoog and Miller, 1957). One simple explanation for the mechanism of action of the topless mutation is that it increases the auxin concentration in apical regions of the embryo.

The activity of the DR5::GUS construct appears to reflect high levels of free auxin in the root (Sabatini et al., 1999). This synthetic promoter consists of seven tandem repeats of an element found in auxin responsive promoters (Ulmasov et al., 1997a). This sequence binds to a family of AUXIN RESPONSE FACTORS (ARF) thought to be involved in transducing the auxin signal. Sabatini et al. (Sabatini et al., 1999) have shown that this reporter is active in the presumptive root in the late heart stage embryo.

If the tpl-1 mutation acts to increase auxin levels in the apical region of the embryo and this, in turn, causes the apical pole to develop root fate, we might expect to see an early and broad accumulation of GUS activity in the apical regions of topless 1; $D R 5: \because G U S$ embryos. To test this hypothesis, we allowed topless 1; DR5::GUS embryos to develop at restrictive temperature $\left(29^{\circ} \mathrm{C}\right)$ and assayed them for GUS expression at 168 hours post-fertilization. While all embryos expressed the reporter in the basal root, only two out of 100 embryos expressed GUS in the developing apical root of the topless mutant embryos at this timepoint. Recall that a much larger proportion of topless embryos express the $\mathrm{J} 1092$ (rootcap) and LENNY reporters by this point (see above). Hundreds of topless;DR5::GUS embryos younger than this stage were examined and none were found to express GUS in the developing apical root. When expression was observed in the apical root, it was found only in small, randomly located, patches (Fig. 6I). Because the DR5::GUS transgene is normally active in the tips of cotyledons later in embryogenesis, we do not know if this patchy accumulation marks cells because they are presumptive root cells or because they are presumptive cotyledon tip cells. In either case, this finding argues against the simple hypothesis that the tpl-1 mutation mediates apical root development by increasing auxin levels in the apical pole of the embryo.

\section{The topless-1 mutation partially suppresses the monopteros mutant phenotype}

The MONOPTEROS gene encodes a member of the ARF family of transcription factors and is thought to be responsible for transducing auxin signals crucial for root development (Hardtke and Berleth, 1998). monopteros mutants fail to develop a root and most of the hypocotyl during embryogenesis.

MONOPTEROS encodes a protein that has a DNA-binding domain at its $\mathrm{N}$ terminus, and a dimerization domain at its $\mathrm{C}$ terminus (Hardtke and Berleth, 1998). Between these two domains is a predicted activation domain. The rootless mutation (Barton and Poethig, 1993) is an EMS-induced allele of MONOPTEROS. PCR amplification of the MONOPTEROScoding sequence in rootless mutants showed that these mutants carry a stop codon at position 594 of the polypeptide [Q594
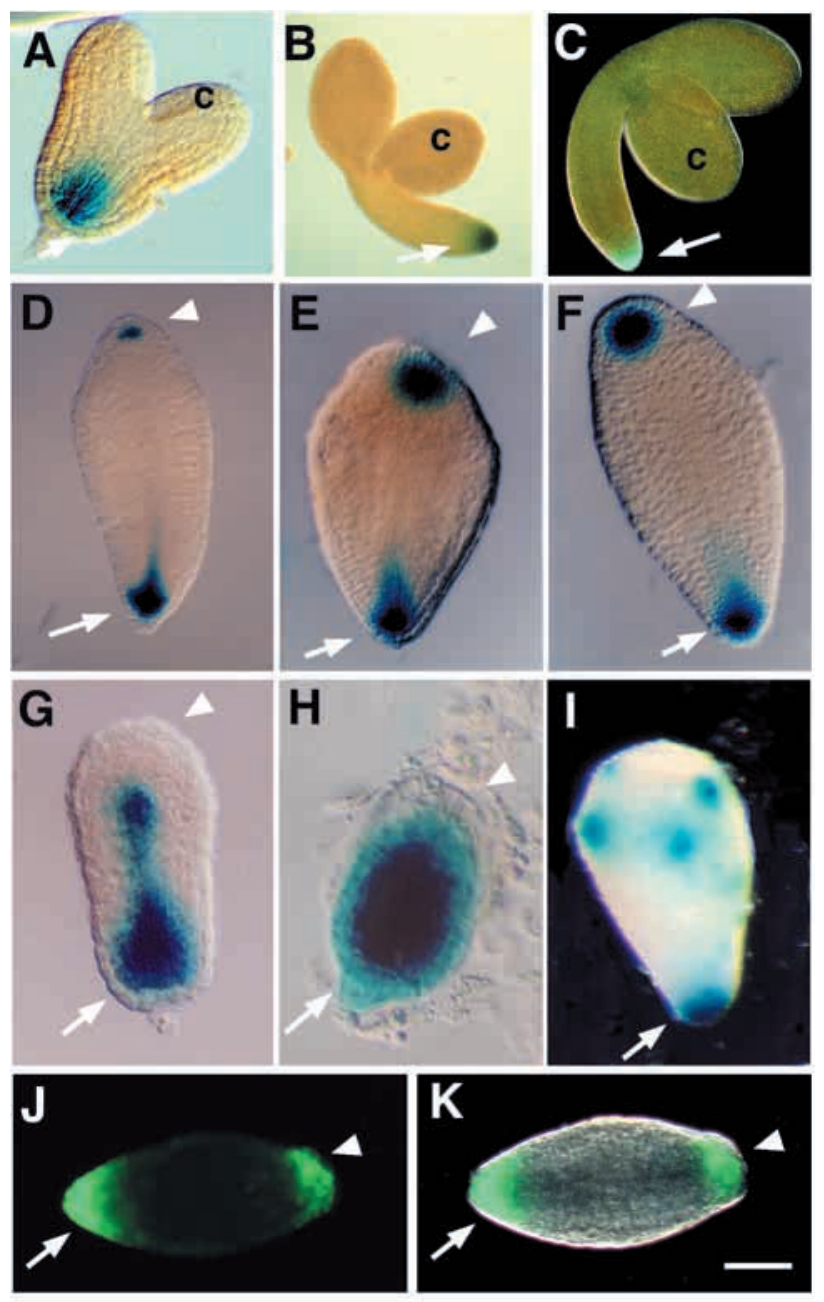

Fig. 6. Expression of the $L E N N Y$ (lenticular cell), DR5 (auxin maximum) and J1092 (root cap) markers in wild-type and topless mutant embryos. (A) Wild-type heart stage embryo expressing the $L E N N Y$ reporter in the lenticular cell and adjacent cells. (B) Wild-type mature stage embryo expressing the $L E N N Y$ reporter. (C) Wild-type mature stage embryo expressing the $J 1092$ reporter expressed in the root cap. (D) topless embryo showing a weak signal for the $L E N N Y$ reporter at the apical pole. (E) topless embryo with $L E N N Y$

expression at both poles. Note that the apical pole is wider and does not yet show root-like cellular organization. (F) topless embryo with $L E N N Y$ expression at both poles. (G) topless embryo arrested as an elongated embryo. Notice the two foci of $L E N N Y$ expression connected to each other. $(\mathrm{H})$ topless embryo arrested at globular stage. $L E N N Y$ reporter is expressed throughout. (I) A rare tpl-1 embryo that expresses the $D R 5$ reporter in the apical region of the embryo. In these rare individuals, expression is typically patchy. (J) Expression of the $J 1092$ root cap marker in a homozygous tpl-1 mutant embryo. (K) Image in J overlaid on Nomarski image of embryo. Scale bars: $25 \mu \mathrm{m}$ in $\mathrm{A}, \mathrm{G}, \mathrm{H} ; 65 \mu \mathrm{m}$ in $\mathrm{B}, \mathrm{C} ; 50 \mu \mathrm{m}$ in D-F,I-K.

(CAG) to STOP (TAG)]. This is downstream of the DNAbinding domain and upstream of the C-terminal dimerization domain. We have renamed this allele monopteros-7 ( $\mathrm{mp}-7)$.

To determine if the topless- 1 mutation acts through the monopteros pathway, we created mutants homozygous for both tpl-1 and $m p-7$ (Fig. 7). Self progeny from a plant of genotype tpl-1 mp-7/tpl-1 MP $\mathrm{Mere}^{+}$scored. (Both genes are located on 

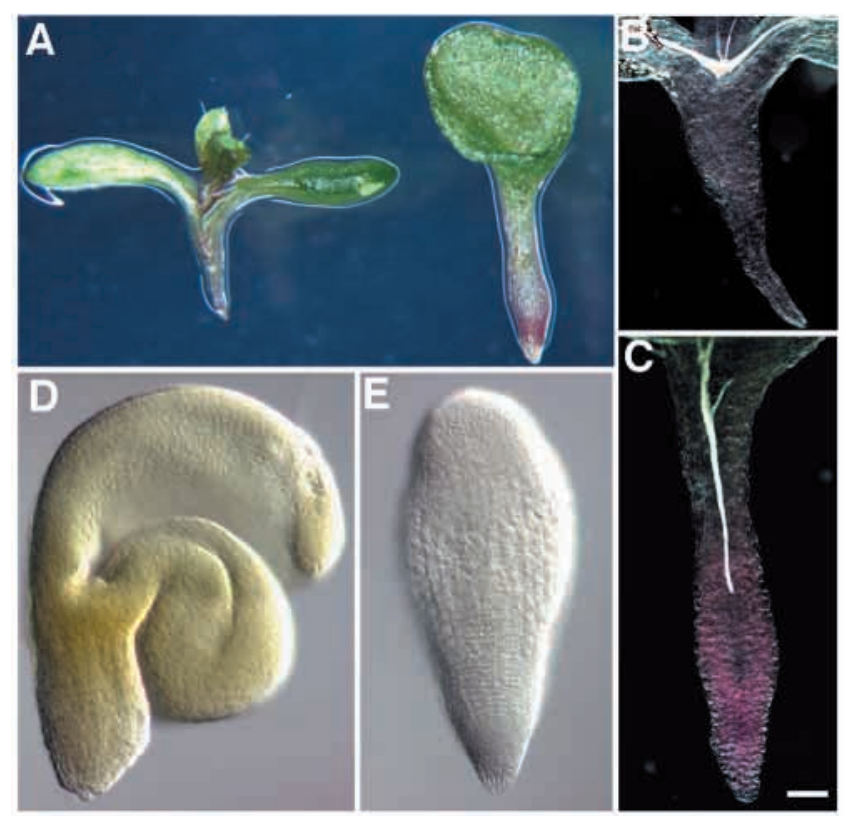

Fig. 7. Phenotypes of mutants homozygous for both tpl-1 and $m p-7$. (A) Seedling to left is $m p-7$ homozygote. Seedling to right is $m p-7$ tpl-1 homozygote. Both seedlings developed at $24^{\circ} \mathrm{C}$. The double mutant has a more well-developed hypocotyl than the $\mathrm{mp}-7$ single mutant. (B) Cleared $m p-7$ single mutant (grown at $24^{\circ} \mathrm{C}$ ) showing lack of vasculature in the hypocotyl 'stub'. (C) Cleared $m p-7$ tpl-1 double mutant (grown at $24^{\circ} \mathrm{C}$ ) showing vasculature extending down into the more well developed hypocotyl. (D) Homozygous $m p-7$ single mutant grown at $29^{\circ} \mathrm{C}$. (E) Homozygous $m p-7$ tpl-1 double mutant grown at $29^{\circ} \mathrm{C}$. The double mutant is able to develop an apical root. Scale bar: $200 \mu \mathrm{m}$ in A; $100 \mu \mathrm{m}$ in B,C; $50 \mu \mathrm{m}$ in D,E.

chromosome 1.) At both 24 and $29^{\circ} \mathrm{C}$, a quarter of the selfprogeny lacked roots and were assumed to be the $t \mathrm{pl}-1 \mathrm{mp}-7 / \mathrm{tpl}$ $1 \mathrm{mp}-7$ double mutants. Thus, the $t p l-1$ mutation did not restore the ability to make a basal root to monopteros mutants. By contrast, the tpl-1 $\mathrm{mp}$-7 double mutants made apical roots similar to $t p l-1$ single mutants (at $29^{\circ} \mathrm{C}$ ). Thus, basal root formation is dependent on wild-type MONOPTEROS activity in topless mutants but apical root formation is not.

The tpl-1 mutation partially suppresses the hypocotyl defect seen in monopteros mutants: $t p l-1 \mathrm{mp}-7$ double mutants were able to form much more hypocotyl tissue than $m p-7$ single mutants (Fig. 7). Whereas only 9\% (8/86) of monopteros single mutants made any detectable vasculature in the basal 'stub' typically found in $\mathrm{mp}$ mutants, 97\% (102/105) of tpl-1 $\mathrm{mp}-7$ mutants showed the presence of hypocotyl-associated vascular development (Fig. 7; this experiment was performed at $24^{\circ} \mathrm{C}$ ). The partial suppression of the monopteros hypocotyl defect by tpl- 1 shows that the tpl-1 mutation can have effects on basal portions of the developing embryo and that mutant tpl-1 activity can partially bypass the requirement for MONOPTEROS activity in the development of the hypocotyl and its vasculature.

\section{Temperature shift experiments show that the $t p l$ mutation can mediate its effects late in embryogenesis}

The temperature sensitivity of the tpl-1 mutation allows for

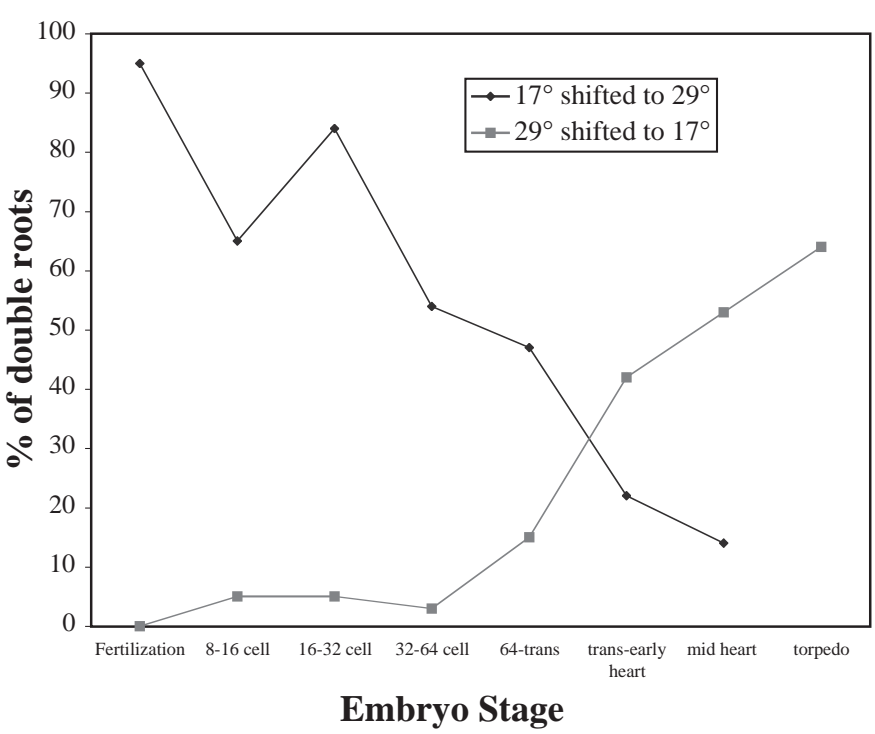

Fig. 8. Effect of temperature shift on topless embryo development. Number of embryos that developed an apical root when shifted from $29^{\circ} \mathrm{C}$ to $17^{\circ} \mathrm{C}$ at fertilization, $0 / 126$; at $8-16$ cells, 6/127; at $16-32$ cells, 8/154; at 32-64 cells, 4/126; at 64 cells to transition stage, 26/175; at transition to early heart stage, 43/103; at midheart stage, 48/91; at torpedo stage, 74/116. Number of embryos that developed an apical root when shifted from $17^{\circ} \mathrm{C}$ to $29^{\circ} \mathrm{C}$ at fertilization, $142 / 149$; at $8-16$ cells, $93 / 143$; at $16-32$ cells, $113 / 134$; at 32-64 cells, 46/85; at 64 cells to transition stage, $92 / 195$; at transition to early heart stage, 31/143; at midheart stage, 18/127.

temperature shift experiments to determine at what point the tpl mutation affects the development of the apical pole of the embryo. For this experiment, homozygous tpl-1 plants were grown at $24^{\circ} \mathrm{C}$. They were then pollinated and placed at $29^{\circ} \mathrm{C}$ or $17^{\circ} \mathrm{C}$. Embryonic stages at the time of shift were determined by dissecting embryos from siliques pollinated and shifted at the same time as the experimental cohort.

Embryos allowed to develop at $17^{\circ} \mathrm{C}$ from fertilization onwards did not develop apical roots (Fig. 8). Embryos that experienced high temperature early in embryogenesis, but developed at $17^{\circ} \mathrm{C}$ from the 32-cell stage and onwards, developed a small fraction of double root seedlings. This indicates that $t p l$ mutant activity, or synthesis of mutant TOPLESS product, during early stages of embryogenesis has the potential to affect development in an irreversible way. A majority of embryos shifted from $29^{\circ} \mathrm{C}$ to $17^{\circ} \mathrm{C}$ at the torpedo stage developed apical roots, indicating that the shift to permissive temperature was often not sufficient to restore normal shoot development after this point.

The experiment in which embryos were shifted from $17^{\circ} \mathrm{C}$ to $29^{\circ} \mathrm{C}$ at varying time points gave largely reciprocal results. Nearly all of the embryos that developed at $29^{\circ} \mathrm{C}$ from fertilization and on developed as double root embryos (Fig. 8). Shifting to $29^{\circ} \mathrm{C}$ during the $16-32$ cell stage also resulted in a majority of double root seedlings, indicating that topless mutant activity or synthesis could alter fates at the globular stage. Surprisingly, shifting embryos from $17^{\circ} \mathrm{C}$ to $29^{\circ} \mathrm{C}$ as late as the transition to midheart stage could cause many of the embryos to make apical roots. This is surprising because transition and heart stage embryos express normal apical 
(shoot) fates not only at the molecular level but also in the development of structures such as cotyledon primordia.

While the results are largely complementary, there are nevertheless differences in the two shift experiments that suggest the embryos are biased toward normal development. For example, an embryo shifted from $29^{\circ} \mathrm{C}$ to $17^{\circ} \mathrm{C}$ from midheart onwards has a roughly $50 \%$ chance of developing cotyledon or SAM fates at the apical pole. However, an embryo shifted from $17^{\circ} \mathrm{C}$ to $29^{\circ} \mathrm{C}$ from midheart on has an $85 \%$ chance of developing cotyledon or SAM fates at the apical pole.

\section{DISCUSSION}

\section{Homozygous topless mutant embryos exhibit transformation of shoot to root at the apical pole}

The tpl-1 mutation causes dramatic changes in the development of the Arabidopsis embryo. The most dramatic and novel topless phenotype is seen at high temperature where embryos develop apical roots in place of shoot apical meristem and cotyledons. The apical root that develops has a recognizable root cap, exhibits normal gravitropism, has root hairs and shows generally normal morphology, although it may be wider than the wild-type root. In agreement with these gross characters, developing tpl- 1 embryos show changes in pattern formation as detected with molecular markers for regional identities.

To our knowledge, this is the first example of a mutation that causes the transformation of apical into basal fates in the embryo of any plant species. The double root phenotype indicates that the $t p l-1$ mutation can bypass the requirement for any basal, root promoting, positional cues laid down in the egg cell or in the early zygote. Analogous mutations that cause transformation of basal into apical fates have not yet been discovered but the existence of the tpl-1 mutation suggests the existence of a master regulatory network that can switch relatively cleanly between apical and basal fates.

At lower temperatures, the seedlings are missing the shoot apical meristem and/or the cotyledons normally found at the apical pole. The SAM is more likely to be missing than are the cotyledons. Consistent with this, expression of the SAM markers $S T M$ and $U F O$ was completely absent in all mutants, even at $24^{\circ} \mathrm{C}$. Thus, $t p l$ mutants are unable to specify SAM identity during embryogenesis. By contrast, topless mutant embryos appear to be able to specify cotyledon identity as ANT expression, although somewhat abnormal in pattern, is present. Surprisingly, $A N T$ was even found to be present in embryos developing at $29^{\circ} \mathrm{C}$, even though most of these embryos develop apical roots in place of cotyledons.

The abnormal pattern of $A N T$ expression in topless mutant embryos, expression throughout the apical dome instead of only in a peripheral ring, indicates that pattern formation is defective not only in the apical-basal dimension but also in the radial dimension.

As loss of STM function is known to cause cotyledon fusion, the cotyledon defects may in part be a result of failure of homozygous tpl-1 seedlings to accumulate STM product. However, the severity of cotyledon fusion in $t p l-1$ seedlings can go well beyond what is seen in stm mutants, indicating that the regulation of other genes must be affected. Candidates include the CUP-SHAPED COTYLEDON 1 and CUP-SHAPED COTYLEDON 2 genes; when simultaneously mutant, cucl and сис 2 cause the development of a cup-shaped cotyledon, failure to express STM and failure to form a SAM (Aida et al., 1997; Aida et al., 1999). This phenotype is seen in some tpl-1 mutants. It will be interesting to find out whether tpl-1 mutants are affected in $C U C$ expression.

The effect of tpl-1 on the development of the hypocotyl is more subtle. With the exception of the double root seedlings, tpl-1 mutant hypocotyls appear superficially normal. However, developing topless embryos show greatly reduced KNAT1 transcript, a transcript normally specific to the developing hypocotyl. (Failure to express KNAT1 by tpl-1 mutant embryos is not a secondary consequence of failure to form a SAM, as stm mutants are able to express KNAT1 (J. L. and M. K. Barton, unpublished). Additional evidence for action of the $t p l-1$ mutation in the region of the developing hypocotyl is seen in the ability of the tpl-1 mutation to partially restore hypocotyl development in monopteros mutants.

\section{Polarity in the embryo remains susceptible to $t p l$ mutant activity until the heart stage}

Temperature-shift experiments of developing topless embryos show that the probability of apical root development can be influenced by temperature even after cotyledons have begun to develop. Thus, a significant number $(20 \%)$ of seedlings will develop apical roots when topless embryos are shifted to restrictive temperature at the transition to early heart stage, a stage when the presumptive cotyledons are beginning to grow out. This result strongly suggests that cells initially developing with apical fates are redirected into root fates in $t p l$ embryos. Several other observations are consistent with this as well. First, ANT, a cotyledon marker, is expressed in all topless embryos even in conditions in which the embryos will develop apical roots. Second, the development of the apical root seems to occur later than that of the primary root in many topless embryos. This is indicated by our finding that despite the fact that a majority of topless embryos develop into double-root seedlings, a minority of topless embryos show expression of root specific molecular markers by 168 hours (7 days) postfertilization. Finally, the $t p l-l$ apical root appears to derive from a larger group of cells than does a normal root. In the embryo, the $t p l$ apical root is morphologically broader than a normal root and lacks the cellular organization of a normal root. Development from a larger group of cells is expected if the apical root develops from the more expanded apical region of a transition stage embryo.

Given the apparent respecification of shoot structures into root structures at the apical pole of at least some topless embryos, it will be interesting to learn whether the shoot to root transformation requires first dedifferentiation followed by redifferentiation, or whether apical cells switch fates directly, without undergoing an intervening stage of dedifferentiation.

One can speculate that the reason the double root embryos survive to be recognized at all is, in fact, because they undergo such a re-specification. If the apical pole of the globular embryo is mis-specified as 'basal' from the outset, the resulting 'double-basal' embryo may fail to develop sufficiently to complete embryogenesis. For example, failure to establish polarity along the apical/basal axis correctly might prevent growth of the embryo. The observation of arrested embryos 
expressing root markers throughout the embryo is one line of evidence for such an effect. However, it will be crucial to determine that this arrest is due to the tpl-1 mutation and not to a second unrelated mutation in this genetic background.

\section{Experiments fail to link the topless 1 mutation to alterations in auxin signaling}

It has long been recognized that a high auxin to cytokinin ratio will promote root development over shoot development in cultured cells (Skoog and Miller, 1957). One possibility is therefore that TPL interferes with the perception of auxin to cytokinin ratios. tpl mutant embryos could have altered distribution patterns of these hormones, increased sensitivity to auxin or reduced sensitivity to cytokinins.

Our results do not support the simple hypothesis that the topless- 1 mutation increases auxin levels in the apical pole of the embryo and thereby causes apical root development. If this were true, we would have expected to see early and ectopic expression of the DR5::GUS reporter; expression of this reporter is believed to reflect high auxin concentrations in the root (Sabatini et al., 1999). Instead, while a significant fraction of topless 1 embryos had begun to differentiate root fates by 168 hours postfertilization (as determined by the J1092 GFP and $L E N N Y$ reporters), only a small fraction had begun to express the DR5::GUS promoter. One problem with the interpretation of this experiment is that the DR5::GUS promoter may not function as a reliable reporter for high free auxin levels in apical regions of the embryo.

The MONOPTEROS gene product has been implicated in auxin action in the developing root (Hardtke and Berleth, 1999). MONOPTEROS is required for the proper development of vasculature in the embryo and for the development of basal (hypocotyl and root) fates during embryogenesis. Many classical studies have indicated that auxin promotes both vascular and root development when exogenously applied. The finding that the MONOPTEROS gene product is a transcription factor that is similar to transcription factors believed to transduce auxin signals is further evidence that MONOPTEROS promotes vascular and root development through an auxin-regulated mechanism.

The MONOPTEROS gene product was not necessary for the formation of the apical root in topless mutant embryos. This is similar to the finding that MONOPTEROS is not required for ectopic root formation from the hypocotyl and cotyledons in superroot mutants (M. K. B., unpublished), the difference being that the apical root in topless mutants forms during embryogenesis, while the ectopic roots in superroot mutants form postembryonically. As there are many ARF-like genes in the Arabidopsis genome, it is possible that one or more homologous genes substitute for MONOPTEROS function when roots are formed from apical regions of the plant. Alternatively, topless may bypass the requirement for ARF-LIKE genes in apical root formation. By contrast, topless could not bypass the requirement for MONOPTEROS in the formation of the basal root.

topless monopteros double mutant individuals make more hypocotyl tissue than monopteros single mutants, indicating that the topless 1 mutation partially suppresses the monopteros phenotype. This could indicate that the tpl- 1 mutation activates a developmental pathway that can substitute in part for MONOPTEROS activity in hypocotyl development. Alternatively, the topless mutation may boost the activity of whatever residual MONOPTEROS activity exists in the monopteros-7 mutant.

In summary, the relationship of topless mutant activity to auxin signaling in root development remains unclear. Preliminary observations of axrl topless double mutants show only additive interactions (J. L., unpublished), indicating that the two processes may indeed be independent of one another. Consistent with the notion of independent action of topless and auxin signaling, treatment of developing Indian mustard embryos with auxin transport inhibitors resulted in ball- or tube-shaped seedlings, but not in double rooted seedlings, as seen in topless mutants (Liu et al., 1993).

An additive action of topless and auxin signaling could also explain the temperature sensitive nature of the $t p l-1$ mutation. Gray and co-workers have described auxin-dependent processes that are enhanced by high temperature (Gray et al., 1998). In addition, they measured higher levels of auxin in plants grown under high temperatures than under low temperatures. Thus, a combination of a mutant $t p l$ gene product plus enhanced sensitivity to auxins and/or higher auxin levels at high temperatures might act in combination to explain the temperature sensitivity of the topless phenotype.

\section{The role of TOPLESS in wild-type embryo development}

The TOPLESS locus is currently represented by only one mutant allele. This allele is temperature sensitive and also shows a weak dominant effect. Additional screens for toplesslike mutants have failed to turn up more alleles of the $T P L$ locus, indicating that the change in tpl-1 is likely to be a rare type of mutation (J. L. and M. K. B., unpublished).

Given the unique nature of this allele, it is difficult to determine the wild-type function of TPL. Several roles are possible, depending on the nature of the tpl-1 mutation. If $t p l$ 1 is a loss-of-function or dominant negative mutation, the wildtype role of tpl-1 is to confer shoot identity (or repress root identity) to the apical pole. However, if the mutation elevates wild-type TOPLESS activity or makes TOPLESS activity spatially or temporally constitutive, the wild-type role would be to confer root fate to the basal pole. Given the difficulty in obtaining new alleles, the molecular cloning of the TOPLESS locus is likely to be the most direct route to determining its wild-type function.

Rare dominant alleles of genes are difficult to obtain except through the use of efficient point mutagens such as EMS. Such alleles provide important clues to gene function that are easily missed in standard reverse genetic studies that rely on 'knockout' or overexpression strategies. Examples of rare dominant mutations that disrupt regulation of protein products are those in the ETRI (ethylene resistant 1) and PHABULOSA genes (Bleecker et al., 1988; Chang et al., 1993; McConnell and Barton, 1998). Interestingly, both of these gene products appear to act as receptors. Whatever role TOPLESS plays in wild-type development, understanding and manipulating the tpl-1 gene should lead to insights in embryonic pattern formation, as well as the ability to manipulate the generation of new root apical meristems from shoot tissue.

This is paper number 3525 from the Laboratory of Genetics. We thank members of the Barton laboratory and Donna Fernandez for helpful comments on the manuscript. We also acknowledge the 
support and generosity of Venkatesan Sundaresan in whose laboratory the work on the LENNY marker was initiated. The work in this manuscript was supported by NSF grant \#IBN-9973579 to M. K. B., a Helen Hay Whitney Postdoctoral Fellowship to J. L. and NSF grant number MCB-9603821 to E. M.

\section{REFERENCES}

Aida, M., Ishida, T., Fukaki, H., Fujisawa, H. and Tasaka, M. (1997). Genes involved in organ separation in Arabidopsis - an analysis of the cupshaped cotyledon mutant. Plant Cell 9, 841-857.

Aida, M., Ishida, T. and Tasaka, M. (1999). Shoot apical meristem and cotyledon formation during Arabidopsis embryogenesis: interaction among the CUP-SHAPED COTYLEDON and SHOOT MERISTEMLESS genes. Development 126, 1563-1570.

Barton, M. K. and Poethig, R. S. (1993). Formation of the shoot apical meristem in Arabidopsis thaliana: an analysis of development in the wild type and in the shoot meristemless mutant. Development 119, 823-831.

Bleecker, A. B., Estelle, M. A., Somerville, C. and Kende, H. (1988). Insensitivity to ethylene conferred by a dominant mutation in Arabidopsis thaliana. Science 241, 1086-1089.

Busch, M., Mayer, U. and Jurgens, G. (1996). Molecular analysis of the Arabidopsis pattern formation of gene GNOM: gene structure and intragenic complementation. Mol. Gen. Genet. 250, 681-691.

Chang, C., Kwok, S. F., Bleecker, A. B. and Meyerowitz, E. M. (1993). Arabidopsis ethylene-response gene ETR1: similarity of product to twocomponent regulators. Science 262, 539-544.

Di Laurenzio, L., Wysocka-Diller, J., Malamy, J. E., Pysh, L., Helariutta, Y., Freshour, G., Hahn, M. G., Feldmann, K. A. and Benfey, P. N. (1996). The SCARECROW gene regulates an asymmetric cell division that is essential for generating the radial organization of the Arabidopsis root. Cell 86, 423-433.

Dolan, L., Janmaat, K., Willensen, V., Linstead, P., Pothig, S., Roberts, K. and Scheres, B. (1993). Cellular organization of the Arabidopsis thaliana root. Development 119, 71-84.

Elliott, R. C., Betzner, A. S., Huttner, E., Oakes, M. P., Tucker, W. Q. J., Gerentes, D., Perez, P. and Smyth, D. R. (1996). AINTEGUMENTA, an APETALA2-like gene of Arabidopsis with pleiotropic roles in ovule development and floral organ growth. Plant Cell 8, 155-168.

Gray, W. M., Ostin, A., Sandberg, G., Romano, C. P. and Estelle, M. (1998). High temperature promotes auxin-mediated hypocotyl elongation in Arabidopsis. Proc. Natl. Acad. Sci. USA 95, 7197-7202.

Hamann, T., Mayer, U. and Jurgens, G. (1999). The auxin-insensitive bodenlos mutation affects primary root formation and apical-basal patterning in the Arabidopsis embryo. Development. 126, 1387-1395.

Hardtke, C. S. and Berleth, T. (1998). The Arabidopsis gene MONOPTEROS encodes a transcription factor mediating embryo axis formation and vascular development. EMBO J. 17, 1405-1411.

Hobbie, L., McGovern, M., Hurwitz, L. R., Pierro, A., Liu, N. Y., Bandyopadhyay, A. and Estelle, M. (2000). The axr6 mutants of Arabidopsis thaliana define a gene involved in auxin response and early development. Development 127, 23-32.
Jürgens, G., Mayer, U., Torres Ruiz, R. A., Berleth, T. and Misera, S. (1991). Genetic analysis of pattern formation in the Arabidopsis embryo. Development Suppl. 1, 27-38.

Konieczny, A. and Ausubel, F. M. (1993). A procedure for mapping Arabidopsis mutations using do-dominant ecotype-specific PCR-based markers. Plant J. 4, 403-410.

Lee, I., Wolfe, D. S., Nilsson, O. and Weigel, D. (1997). A LEAFY coregulator encoded by UNUSUAL FLORAL ORGANS. Curr. Biol. 7, 95104.

Lincoln, C., Long, J., Yamaguchi, J., Serikawa, K. and Hake, S. (1994). A knotted1-like homeobox gene in Arabidopsis is expressed in the vegetative meristem and dramatically alters leaf morphology whan overexpressed in transgenic plants. Plant Cell 6, 1859-1876.

Liu, C. M., Xu, Z. H. and Chua, N. H. (1993). Auxin polar transport is essential for the establishment of bilateral symmetry during early plant embryogenesis. Plant Cell 5, 621-630.

Long, J. A. and Barton, M. K. (1998). The development of apical embryonic pattern in Arabidopsis. Development 125, 3027-3035.

Long, J. A., Moan, E. I., Medford, J. and Barton, M. K. (1996). A member of the KNOTTED class of homeodomain proteins encoded by the SHOOTMERISTEMLESS gene of Arabidopsis. Nature 379, 66-69.

Mansfield, S. G. and Briarty, L. G. (1991). Early embryogenesis in Arabidopsis thaliana. II. The developing embryo. Can. J. Bot. 69, 447-476.

Mansfield, S. G., Briarty, L. G. and Erni, S. (1991). Early embryogenesis in Arabidopsis thaliana. I. The mature embryo sac. Can. J. Bot. 69, 447460.

Mayer, U., Buttner, G. and Jürgens, G. (1993). Apical-basal pattern formation in the Arabidopsis embryo: studies on the role of the gnom gene. Development 117, 149-162.

McConnell, J. and Barton, M. K. (1998). Leaf polarity and meristem formation in Arabidopsis thaliana. Development 125, 2935-2942.

Sabatini, S., Beis, D., Wolkenfelt, H., Murfett, J., Guilfoyle, T., Malamy, J., Benfey, P., Leyser, O., Bechtold, N., Weisbeek, P. and Scheres, B. (1999). An auxin-dependent distal organizer of pattern and polarity in the Arabidopsis root. Cell 99, 463-472.

Shevell, D. E., Leu, W. M., Gillmor, C. S., Xia, G., Feldmann, K. A. and Chua, N. H. (1994). EMB30 is essential for normal cell division, cell expansion, and cell adhesion in Arabidopsis and encodes a protein that has similarity to Sec7. Cell 77, 1051-1062.

Skoog, F. and Miller, C. O. (1957). Chemical regulation and organ formation in plant tissues cultured in vitro. Symp. Soc. Exp. Biol. 11, 118-131.

Steinmann, T., Geldner, N., Grebe, M., Mangold, S., Jackson, C. L., Paris, S., Galweiler, L., Palme, K. and Jurgens, G. (1999). Coordinated polar localization of auxin efflux carrier PIN1 by GNOM ARF GEF. Science 286, 316-318.

Sundaresan, V., Springer, P., Volpe, T., Haward, S., Jones, J. D., Dean, C., Ma, H. and Martienssen, R. (1995). Patterns of gene action in plant development revealed by enhancer trap and gene trap transposable elements. Genes Dev. 9, 1797-1810.

Ulmasov, T., Hagen, G. and Guilfoyle, T. J. (1997). ARF1, a transcription factor that binds to auxin response elements. Science 276, 1865-1868.

Vernon, D. M. and Meinke, D. W. (1994). Embryogenic transformation of the suspensor in twin, a polyembryonic mutant of Arabidopsis. Dev. Biol. 165, 566-573. 